\title{
2-Metoksipiridin-3-Boronik Asitin Lineer Olmayan Özellikleri, Konformasyonel, Titreşimsel ve Elektronik Yapısı Üzerine Substitüent Etkisinin Kuantum Mekanik Metodlar ile Araştırılması
}

\author{
Güventürk UĞURLU(iD
}

Kafkas Üniversitesi, Fizik Bölümü, 36100 Kars, Türkiye

Geliş / Received: 18/04/2018, Kabul / Accepted: 04/01/2019

\begin{abstract}
$\ddot{O} \mathbf{z}$
Bu çalışmada, 2-metoksipiridin-3-boronik asit (I) ve 6-sübstitüe-2-metoksipiridin-3-boronik asit türevleri; 6floro-2-metoksipiridin-3-boronik (II) ve 6-kloro-2-metoksipiridin-3- boronik asit (III) moleküllerinin yapisal parametreleri, titreşim frekansları, dipol moment $(\mu)$, polarizebilite $(\alpha)$ ve hiperpolarizebilite $(\beta)$ değerleri Hartree Fock (HF) ve Yoğunluk Fonksiyonel Teorisi (DFT/B3LYP) metotlarında 6-311 ++ G (d, p) temel seti kullanılarak hesaplatıldı. I, II ve III moleküllerinin en yüksek dolu molekül orbital (HOMO) ve en düşük boş molekül orbital (LUMO) aynı metot-temel seti kombinasyonu ile hesaplandı ve enerji aralıkları ( $\Delta \mathrm{Eg}$ ) incelendi. ${ }^{1} \mathrm{H}$ ve ${ }^{13} \mathrm{C}$-NMR kimyasal kayma değerleri GIAO yaklaşımına göre gaz fazında B3LYP/6-311+G (2d,p) ve HF/6-31G (d) yöntemleri ile hesalandı. Ayrıca, moleküllerin potansiyel enerji yüzeyi (PEY), C1-C2-B-O1 dihedral açısının fonksiyonu olarak her iki metotta $6-31+\mathrm{G}$ temel seti kullanılarak yapıldı. Hesaplanan PEY üzerinde $0^{\circ}, 140^{\circ}, 220^{\circ}$ ve $360^{\circ}$ de minimum, $90^{\circ}, 180^{\circ}$ ve $270^{\circ}$ de ise maksimumlar yer almaktadır. Moleküllerin hiperpolarizebilite değerlerinin sıralaması III $>\mathbf{I I}>\mathbf{I}$ şeklindedir. En büyük bariyer yüksekliğine $180^{\circ}$ de I molekülünün sahip olduğu görüldü. I, II ve III moleküllerinin dipol moment değerleri sirasiyla, B3LYP / 6-311 ++ G (d, p) metot-temel seti kombinasyonu ile 1.18, 1.19 ve1.25 HF / 6-311 ++ G (d, p) metot-temel seti kombinasyonu 1.17, 1.16 ve 1.30 Debye bulundu. Her iki metotta hesaplanan I molekülünün yapısal parametreleri, literatürdeki verilerle karşılaştırıldı ve yapısal parametreler arasında iyi bir uyum olduğu görüldü.
\end{abstract}

Anahtar Kelimeler: 2-metoksipiridin-3-boronik asit, Polarizebilite, HF, DFT / B3LYP, $\Delta$ Eg

Investigation of Substitute Effect on Non-Linear Properties, Conformational, Vibrational and Electronic Structure of 2-Methoxypyridine-3-Boronic Acid by Quantum Mechanical Methods

\begin{abstract}
In this study, the values of structural parameters, vibration frequencies, dipole moment $(\mu)$, polarizability $(\alpha)$, hyperpolarizability $\beta$ ) of 2-methoxypyridine-3-boronic acid (I) and 6-substituted-2-methoxypyridine-3-boronic acid derivatives; 6-fluoro-2-methoxypyridine-3-boronic (II) and 6-chloro-2-methoxypyridine-3-boronic acid (III) molecules have been calculated at Hartree Fock (HF) and Density Functional Theory (DFT / B3LYP) with 6-311++G (d, p) basis set. The highest occupied molecular orbital (HOMO), the lowest unoccupied molecular orbital (LUMO) of I, II ve III molecules have been computed and their respective gap $(\Delta \mathrm{Eg})$ have been examined. The $1 \mathrm{H}$ and $13 \mathrm{C}$ NMR chemical shift values were calculated in gas phase by GIAO approach using B3LYP/6-311+G (2d,p) and HF/6-31G (d) level of theory. In addition, the potential energy surface (PES) of the molecules as a function of the dihedral angle (C1-C2-B-O1) have been carried out using the 6-31+G basis set in both methods. There are minimums at $0^{\circ}, 140^{\circ}, 220^{\circ}$ and $360^{\circ}$, maximums at $90^{\circ}, 180^{\circ}$ and $270^{\circ}$ on the calculated potential energy surfaces. The order of the hyperpolarizability values of the molecules is III $>$ II $>\mathbf{I}$. It was seen that I molecule had at the maximum barrier height at $180^{\circ}$. The dipole moment values of molecules I, II and III are found as 1.18, 1.19 and 1.25 at B3LYP / 6-311++G (d, p) method-basis set combination and $1.17,1.16$ and 1.30 Debye at HF / 6-311++G (d, p) method-basis set combination, respectively. The structural parameters of the I molecule, which were calculated by both methods, were compared with the data in the literature and there was a good agreement between the structural parameters.
\end{abstract}

Keywords: 2-methoxypyridine, 3-boronic acid, polarizability, HF, DFT / B3LYP, $\Delta$ Eg 


\section{Giriş}

Genellikle boronik asitler yapisal olarak R-B $(\mathrm{OH})_{2},(\mathrm{R}=$ alkil-, alkenil-, alkinil- ve aril) formunda temsil edilirler. Bor tabiatta doğal olarak bulunmamasına rağmen onunla ilgili çalışmalar 1860 yılından beri aralıksız olarak devam etmektedir (Frankland et al. 1860). Boronik asitlerin reaksiyon kabiliyeti ve çok yönlü reaktivitesi gibi önemli özelliğe sahip olması onun kimyasal ve fiziksel özelliklerine dayanır. Üç değerlikli bor atomunun elektron yapısı $\mathrm{sp}^{2}$ hibritleşmesine yatkın olup, boş porbitaline sahiptir. Bundan dolayı bor atomu trigonel $\mathrm{sp}^{2}$ hibritleşmesinden tetrahedral $\mathrm{sp}^{3}$ hibritleşme formuna kolayca dönüşebilmektedir. Nükleofiller, borun boş porbitali ile etkileşime girerek hibritleşmenin türünde ve şeklinde değişime yol açabilmektedir. Boronik asit ve türevleri ile yapılan çalışmalarda bu bileşikler, kristal mühendisliği (Varughese et al., 2011), malzeme bilimi (Petasis, 2007; Cannizzo et al., 2005), tıbbi bilimler (Yang et al., 2003; Baker et al., 2006), biyoorganik (Cooper et al., 1998; Jabbour et al., 2004) ve kimyasal biyoloji (Halo et al., 2009; Yang et al., 2004) gibi bir çok alanda kullanılmaktadır. Suzukitipi çapraz kenetlenme reaksiyonlarının yaygın uygulanması Heterosiklik boronik asit ve türevlerinin sentezlenmesinde ve kullanılmasında hızlı bir büyüme sağlamıştır. Heterosiklik boronik asit ve esterlerinin bir alt grubu olan piridinilboronik asit ve esterleri de ilaç tasarımında kullanıldıklarından dolayı büyük öneme sahiptir. Piridinil türevlerinin Suzuki-tipi çapraz kenetlenme reaksiyonlarında nükleofilik olarak işlem yapmasına rağmen bu konu ile ilgili yayın sinırlidir (Bouillon et al., 2002; Cai et al., 2002). Yaygin olarak, piridinilboronik asitlerin çoğu piridin halkası üzerinde yalnız bir boronik asit substitüentine sahiptir. 3piridinilboronik asit ve 4-piridinilboronik asitlerin ilk sentezlerinden biri 1965 yllında yapılmışıtır (Fischer et al., 1965). Sentezlenen bu bileşiklerin kimyasal ve fiziksel özellikleri araştırlarak, termal özellikleri Fischer ve arkadaşları tarafından belirlenmiştir (Fischer et al., 1974). Bu çalışmada, 2-metoksipiridin3-boronik asit (I) ve 6-substitüe-2metoksipiridin-3-boronik asit türevleri olan 6floro-2-metoksipiridin-3-boronik (II) ve 6kloro-2-metoksipiridin-3-boronik asit (III) moleküllerinin yapısal parametreleri, titreşim frekansları, $\mu, \alpha, \beta, \mathrm{E}_{\text {номо }}, \mathrm{E}_{\mathrm{LUMO}}$ ve $\Delta \mathrm{E}_{\mathrm{g}}=$ $\mathrm{E}_{\mathrm{LUMO}} \mathrm{E}_{\text {Hомо }}$ değerleri DFT/B3LYP ve HF metodları ile farklı taban setleri kullanılarak hesaplanmıştır. $\mathrm{Bu}$ molelüllerden, I molekülünün yanlızca yapısal parametreleri Dabrowski ve arkadaşları tafafindan (Dabrowski et al., 2006), III molekülünün ise ${ }^{1} \mathrm{H}-$ ve ${ }^{13} \mathrm{C}-\mathrm{NMR}$ kimyasal kayma değerleri Saygil1 tarafindan deneysel olarak belirlenmiş̧tir (Saygil1, 2011). II molekülü ilk olarak bu çalışmada modellenerek I, ve III molekülleri ile birlikte moleküler özellikleri teorik olarak detaylı bir şekilde incelenmiștir. Belirlenen bu önemli moleküler özellikler I, II ve III ile yapılacak yeni çalışmalara yardımcı olacaktır. Bu çalışmada incelenen moleküllerin numaralandırılmıș kimyasal yapısı Şekil 1'de verilmiştir.

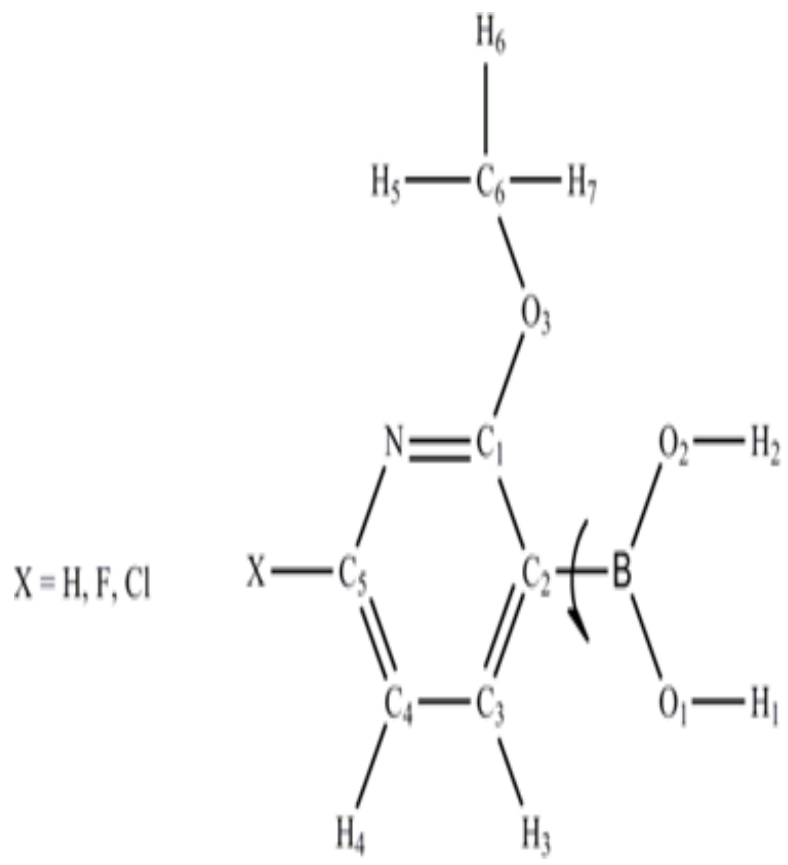

Şekil 1. 6-sübstitüe-2-metoksipiridin-3-boronik asidin numaralandırılmış yapısı. 


\section{Materyal ve Method}

$\mathrm{Bu}$ çalışmada, 2-metoksipiridin-3-boronik asit (I), 6-floro-2-metoksipiridin-3-boronik (II) ve 6-kloro-2-metoksipiridin-3-boronik asit (III) moleküllerinin üç boyuttaki (3D) yaklaş1k geometrisi Gauss View5.0 (Dennington et al., 2009) görüntüleme paket programında çizilerek Gaussian09 Rev B.01 paket programına (Frisch et al., 2010) giriş verisi olarak kullanıldı. I, II ve III moleküllerinin en düşük enerjili konformasyonlarını belirlemek için, moleküllerin elektronik enerjileri C1-C2-B$\mathrm{O} 2$ torsiyon açısının fonksiyonu olarak hem ab-initio metodu HF (Moller et al., 1934) hem de DFT/Becke'nin 3 parametreli hibrit değiştokuş fonksiyoneli (B3) (Becket et al., 1988) ile Lee-Yang ve Parr'in korelasyon fonksiyonelinden (Lee et al., 1988; Beckel, 1993) oluşan B3LYP teori seviyelerinde 6$31+\mathrm{G}$ temel seti kullanılarak hesaplatıld 1 . Konformasyon analizi yapıldiktan sonra bulunan minimum enerjili geometrik yapılar B3LYP/6-311++G(d,p) ve HF/6$311++\mathrm{G}(\mathrm{d}, \mathrm{p})$ metotları ile simetri sinırlamasi olmaksızın optimize edilmiştir. Hesaplamalardan elde edilen optimize yapilar kullanılarak, moleküllerin denge durumları için her iki modelde titreşim frekansları, $\mu, \alpha$, $\beta, \mathrm{E} \_\mathrm{HOMO}, \mathrm{E} \_\mathrm{LUMO}$ ve $(\Delta \mathrm{Eg}=\mathrm{ELUMO}$ - EHOMO) hesaplandi. Ayrica, elde edilen optimize yapı ile GIAO yöntemine göre $1 \mathrm{H}$ NMR ve 13C-NMR kimyasal kayma değerleri B3LYP/6-311+G(2d,p) ve HF/6-31G teori düzeylerinde (McLean et al., 1980; Krishnan et al., 1980) hesapland. Hesaplamalar sonucunda; polarizebilite ve hiperpolarizebilite değerlerinin kartezyen bileşenleri (a.u) elde edildi. Bu kartezyen bileşen değerlerinden polarizebilite;

$\alpha=\left(\frac{1}{3}\right)\left(\alpha_{x x}+\alpha_{y y}+\alpha_{z z}\right)$

Hiperpolarizebilite; $\beta$

$\beta=\left[\left(\beta_{x x x}+\beta_{x y y}+\beta_{x z z}\right)^{2}+\left(\beta_{y y y}+\beta_{y z z}+\right.\right.$

$\left.\left.\beta_{y x x}\right)^{2}+\left(\beta_{z z z}+\beta_{z x x}+\beta_{z y y}\right)^{2}\right]^{1 / 2} \quad$ [2]

Eşitlikleri ile hesaplandi.

\section{Bulgular}

\subsection{Konformasyonel analiz}

Konformasyon analizi ile molekülün toplam potansiyel enerjisini minimum yapan molekül yapısı bulunur. Bir potansiyel enerji yüzeyi (PEY), molekülün geometrisinin bir fonksiyonu olarak molekülün enerjisini veren matematiksel bir fonksiyondur:

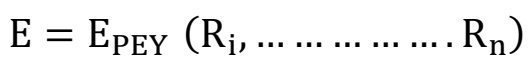

Burada $\mathrm{R}_{\mathrm{i}}, \mathrm{N}$ atomlu doğrusal olmayan bir molekül sisteminin potansiyel enerji yüzeyi $3 \mathrm{~N}-6$, doğrusal molekül için ise $3 \mathrm{~N}-5$ tane koordinat boyutuna sahip olacaktır. Bu çalışmada I, II ve III moleküllerinin konformasyon analizi, C1-C2-B-O2 dihedral açısını $10^{\circ}$ aralıklarla 36 adım değiştirilerek potansiyel enerji eğrileri hesaplatıldı. I, II ve III moleküllerinin, C1-C2-B-O2 dihedral açısının fonksiyonu olarak hesaplanan potansiyel enerji eğrileri Şekil 2(a) ve (b)'de verilmiştir. Şekil 2'de görüldüğü gibi, potansiyel enerji eğrisi üzerinde $0^{\circ}, 140^{\circ}, 220^{\circ}$ ve $360^{\circ}$ de minimum $90^{\circ}, 180^{\circ}$ ve $270^{\circ}$ de ise maksimumlar yer almaktadır. Bu sonuçlardan, çalışılan moleküllerin potansiyel enerji eğrilerinin benzer davranışlar sergilediği görülmektedir. 2-metoksipiridin-3-boronik asit (I), 6-kloro-2-metoksipiridin-3-boronik asit (III) ve 6-floro-2-metoksipiridin-3boronik (II) moleküllerinin $180^{\circ}$ deki bariyer yüksekliği sırasıyla B3LYP metodunda 8.47 , 8.18 ve 8.11 eV HF' da 9.08, 8.88 ve $8.77 \mathrm{eV}$ olarak belirlendi. Moleküllerin bariyer yükseklik değerleri sıralaması I $>$ III $>$ II şeklindedir. HF yöntemiyle hesaplanan bariyer $\left(180^{\circ}\right.$ deki) yükseklikleri B3LYP metodundan daha yüksektir. Bu fark I, II ve III molekülleri için sırası ile 0.61, 0.66 ve $0.70 \mathrm{eV}$ olarak hesaplandi. 

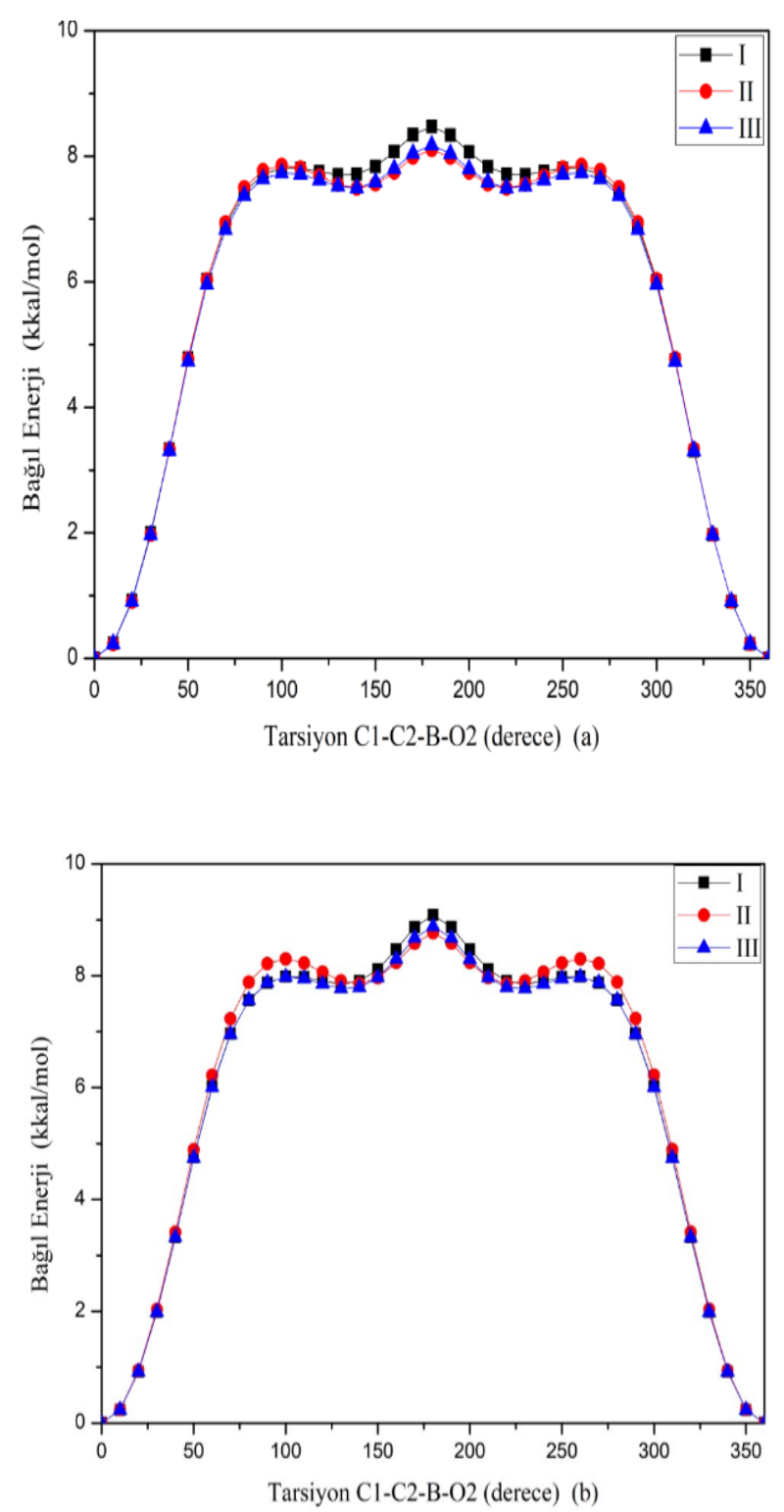

Şekil 2. I,II ve III molekülünün potansiyel eğrisi (a) B3LYP/6-31+G ,(b) HF/6-31+G.

\subsection{Geometrik Optimizasyon}

Konformasyon analizi yapıldiktan sonra bulunan minimum enerjili geometrik yap1 B3LYP/6-31G(d,p) ve HF/6-31G(d,p) metot ile simetri sınırlaması olmaksızın optimize edilmiştir. Çalışılan moleküllerin, optimizasyon sonuçlarından elde edilen geometrik yap1 parametreleri, I molekülün deneysel sonuçları (Dabrowski et al., 2006) ile karşılaştırmalı olarak Tablo 1'de, optimizasyon sonucunda bulunan moleküler yapıları Şekil 3.'de verilmiştir.Çalışılan moleküllerde, optimizasyon hesaplamaları sonucunda moleküllerin optimize durumlarının trans-cis konformerinde olduğu Şekil 2'de görülmektedir. Ancak bu moleküller $-\mathrm{B}(\mathrm{OH})_{2}$ grubundaki hidrojen atomlarının piridin halkası ve onun üzerinde bulunana azot atomuna yönelimlerine göre dört konformasyon durumunda olabilirler (Fragoso-Medina et al., 2017). Molekülde, trans-cis konformeri durumunda azot atomu tarafinda bulunan boronik asit grubundaki hidrojen atomu azot atomuna, diğeri ise piridin halkasından dışa doğru yönelmesidir. $\mathrm{Bu}$ durumun tersi cis-trans konformerine karşılık gelmektedir fakat C3/C1-C2-B$\mathrm{O} 2 / \mathrm{O} 1$ dihedral açılarının C2-B bağı etrafında dönmesi sonucu bu iki konformer birbirine dönüşe bilmektedir. Diğer iki konformer durumu, $\quad-\mathrm{B}(\mathrm{OH})_{2}$ grubundaki hidrojen atomlarının piridin halkasına doğru yönelimi cis-cis tersi durumda trans-trans konformer durumlarını belirlemektedir. N1-C1-O3-C6, C1-C2-B-O1 ve C1-C2-B-O2 dihedral aç1ları B3LYP/6-311++G(d,p) metodunda (I molekül için, sirasıyla $-0.01,179.99,-0.01^{\circ}$, II için $0.02,179.98,-0.01^{\circ}$, III için -0.00 , $\left.180.00, \quad 0.01^{\circ}\right) \quad$ ) $\mathrm{HF} / 6-311++\mathrm{G}(\mathrm{d}, \mathrm{p})$ metodunda (I molekül için, sırasıyla -0.01 , $180.00,0.00^{\circ}$, II için $-0.02,179.99,-0.02^{\circ}$, III için $-0.01,-179.97,0.03^{\circ}$ ) değerleri elde edilmiştir. $\mathrm{Bu}$ değerler her üç molekülünde düzlemsel bir yapıda olduğunu göstermektedir. Ancak, I molekülünün N1C1-O3-C6 dihedral açısını deneysel değeri $3.65^{\circ}$ dir (Dabrowski et al., 2006). Bu kadarlık bir sapmanın deneysel çalışmalarda yapı aydınlatmaları katı fazda yapılır iken, teorik çalışmalarda ise gaz fazında yapılmasından kaynaklandığı düşünülmektedir. Ayrıca, teorik çalışmalarda molekül izole bir ortama sahip iken deneysel çalışma ortamları izole değildir. Moleküllerin dihedral açı değerlerinin hemen hemen aynı olduğu Tablo 1 'de görülmektedir. $\mathrm{Bu}$ da flor ve klor substitüentlerinin dihedral açıları üzerinde etkili olmadığını anlamına gelmektedir. 
Tablo 1. I, II ve III moleküllerinin yapısal parametreleri.

\begin{tabular}{|c|c|c|c|c|c|c|c|}
\hline & \multicolumn{3}{|c|}{ B3LYP/6-311++G (d,p) } & \multirow{2}{*}{\multicolumn{4}{|c|}{$\begin{array}{l}\text { Deneysel }^{\text {a. }} \quad \text { HF/6-311++G }(\mathbf{d}, \mathbf{p}) \\
\text { Bağ uzunluğu/Å }\end{array}$}} \\
\hline & & & & & & & \\
\hline & $\mathrm{X}=\mathrm{H}$ & $\mathrm{X}=\mathrm{F}$ & $\mathrm{X}=\mathrm{Cl}$ & $\mathrm{I}$ & $\mathrm{X}=\mathrm{H}$ & $\mathrm{X}=\mathrm{F}$ & $\mathrm{X}=\mathrm{Cl}$ \\
\hline $\mathrm{C} 1-\mathrm{C} 2$ & 1.4145 & 1.4109 & 1.4112 & & 1.4091 & 1.4028 & 1.4108 \\
\hline $\mathrm{C} 1-\mathrm{N} 1$ & 1.322 & 1.3277 & 1.3261 & & 1.3038 & 1.3121 & 1.3139 \\
\hline $\mathrm{C} 1-\mathrm{O} 3$ & 1.3654 & 1.3589 & 1.3593 & $1,3612(11)$ & 1.3401 & 1.3332 & 1.3184 \\
\hline $\mathrm{C} 2-\mathrm{C} 3$ & 1.3956 & 1.3992 & 1.3973 & & 1.3827 & 1.389 & 1.3853 \\
\hline $\mathrm{C} 2-\mathrm{B}$ & 1.5695 & 1.5675 & 1.5687 & $1,5763(13)$ & 1.5745 & 1.5717 & 1.5696 \\
\hline $\mathrm{C} 3-\mathrm{C} 4$ & 1.3961 & 1.3929 & 1.3939 & & 1.3927 & 1.386 & 1.3897 \\
\hline $\mathrm{C} 4-\mathrm{C} 5$ & 1.3864 & 1.384 & 1.3875 & & 1.3724 & 1.3726 & 1.3722 \\
\hline $\mathrm{C} 5-\mathrm{N} 1$ & 1.3415 & 1.3143 & 1.3215 & & 1.3298 & 1.3029 & 1.3097 \\
\hline $\mathrm{C} 5-\mathrm{X}$ & 1.0861 & 1.3459 & 1.7604 & & 1.0763 & 1.3116 & 1.7417 \\
\hline C6-O3 & 1.437 & 1.4393 & 1.4392 & $1,4408(11)$ & 1.4145 & 1.4176 & 1.4117 \\
\hline O1-B & 1.3703 & 1.3699 & 1.3694 & $1.3533(12)$ & 1.3581 & 1.3578 & 1.3668 \\
\hline $\mathrm{O} 2-\mathrm{B}$ & 1.3642 & 1.3644 & 1.364 & $1.3641(12)$ & 1.3516 & 1.3519 & 1.3538 \\
\hline \multicolumn{8}{|c|}{ Bağ açısi $/^{\circ}$} \\
\hline $\mathrm{C} 2-\mathrm{C} 1-\mathrm{O} 3$ & 116.91 & 117.64 & 117.56 & $116,40(8)$ & 116.99 & 117.77 & 118.26 \\
\hline C1-C2-B & 123.97 & 124.00 & 124.03 & $124,58(8)$ & 124.50 & 124.62 & 125.93 \\
\hline C3-C2-B & 120.96 & 121.01 & 121.07 & & 120.37 & 120.50 & 119.10 \\
\hline $\mathrm{C} 1-\mathrm{O} 3-\mathrm{H} 5$ & 118.10 & 118.27 & 118.21 & & 119.34 & 119.64 & 119.75 \\
\hline B-O1-H2 & 112.02 & 112.16 & 112.17 & & 112.93 & 113.07 & 117.04 \\
\hline B-O2-H1 & 110.98 & 111.34 & 111.41 & & 112.91 & 113.26 & 116.47 \\
\hline C2-B-O1 & 117.55 & 117.41 & 117.38 & $117,028()$ & 117.31 & 117.18 & 115.65 \\
\hline C2-B-O2 & 123.65 & 123.74 & 123.68 & $122,57(8)$ & 124.27 & 124.34 & 122.19 \\
\hline \multicolumn{8}{|c|}{ Torsiyon aç1/ ${ }^{\circ}$} \\
\hline N1-C1-O3-C6 & -0.01 & 0.02 & 0.00 & $-3,65(12)$ & -0.01 & -0.02 & -0.01 \\
\hline C1-C2-B-O1 & 179.99 & 179.98 & 180.00 & & 180.00 & 179.99 & -179.97 \\
\hline $\mathrm{C} 1-\mathrm{C} 2-\mathrm{B}-\mathrm{O} 2$ & -0.01 & -0.01 & 0.01 & & 0.00 & -0.02 & 0.03 \\
\hline C3-C2-B-O1 & -0.01 & -0.02 & 0.01 & & -0.01 & 0.00 & 0.03 \\
\hline $\mathrm{C} 3-\mathrm{C} 2-\mathrm{B}-\mathrm{O} 2$ & -180.00 & 179.98 & 180.00 & & -180.00 & 179.99 & -179.96 \\
\hline $\mathrm{C} 2-\mathrm{B}-\mathrm{O} 2-\mathrm{H} 2$ & 0.00 & 0.00 & 0.00 & & 0.00 & 0.00 & 0.00 \\
\hline C2-B-O1-H1 & 180.00 & 180.00 & 180.00 & & 180.00 & 180.00 & 180.00 \\
\hline
\end{tabular}

a. Dabrowski et al., 2006

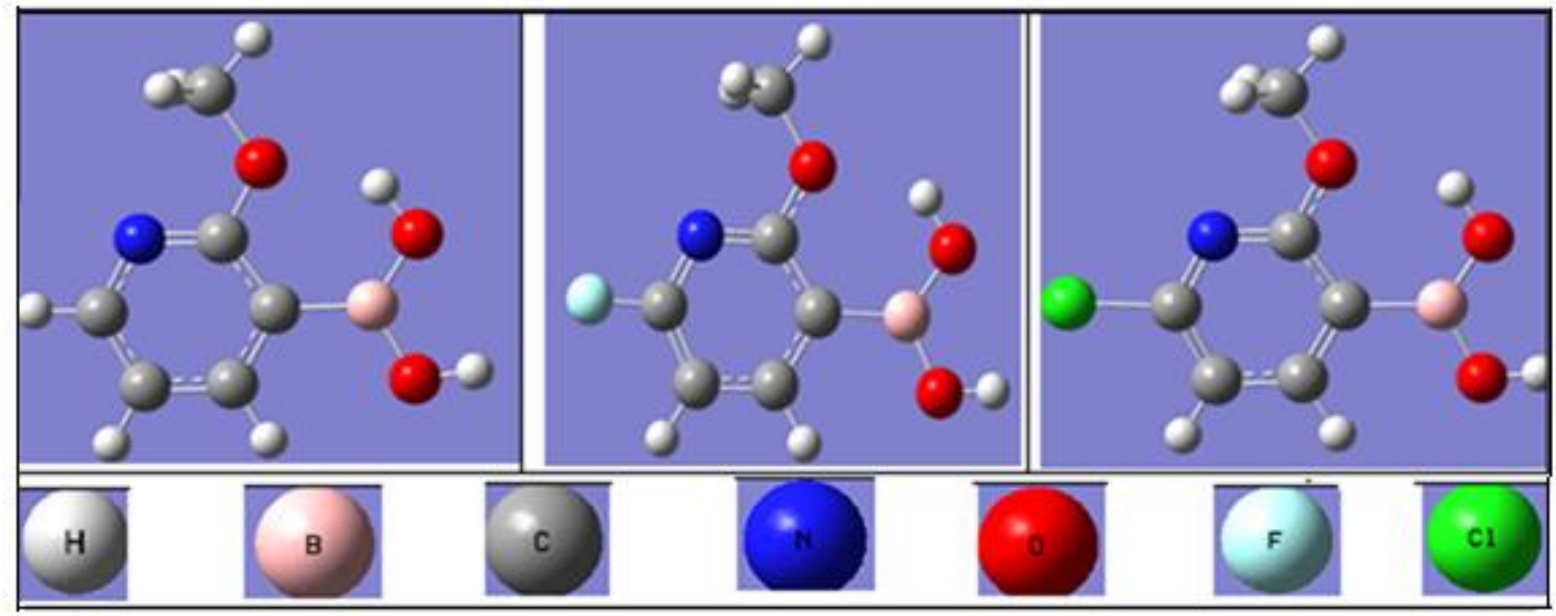

Şekil 3. I,II ve III moleküllerinin optimize geometrileri/B3LYP/6-31G(d,p) 
Literatürde, deneysel olarak yapılan çalışmada, I molekülünün C1-O3, C6-O3 bağ uzunlukları sirasıyla 1.3612 (11) $\AA, 1.4408$ (11) $\AA$ ve C2-C1-O3 bağ açısı ise $116.40^{\circ}$ (8) olarak verildi (Dabrowski et al., 2006). Bu çalışmada, I molekülünün $\mathrm{C} 1-\mathrm{O} 3$ bağ uzunluğu sırası ile 1.3654 ve $1.3401 \AA$ B3LYP/6-311++G(d,p)/ HF/6-311++G(d,p) tespit edilmiştir. I, II ve III moleküllerinin C6-O3 bağ uzunluğu B3LYP/6-311++G(d,p )' de $1.4370,1.4393,1.4392, \AA), \mathrm{HF} / 6-$ $311++\mathrm{G}(\mathrm{d}, \mathrm{p})$ ' de ise $1.4145,1.4176$ ve 1.4117 Å olarak elde edildi. Çalışılan moleküllerin C2-C1-O3 bă̆ açısı sirası ile B3LYP/6$311++\mathrm{G}(\mathrm{d}, \mathrm{p}){ }^{\prime}$ de $116.91,117.64,117.56^{\circ}$, HF/6-311++G(d,p)' de 116.99, 117.77, $118.26^{\circ}$ olarak tespit edildi. Hesaplanmış bağ uzunlukları ile literatürdeki deneysel sonuçlar arasında uyum olduğu gözlendi. Flor ve klor substitüentlerinin bağ açıları üzerinde etkili olduğu ve bu etkinin C2-B-O1ve C2-B-O2 bağ açılarına nispeten $\mathrm{C} 2-\mathrm{C} 1-\mathrm{O} 3$ bağ açıs1 üzerinde daha fazla olduğu tespit edildi.

\subsection{HOMO-LUMO Enerjileri ve Lineer Olmayan Özelliklerinin İncelenmesi}

I, II ve III moleküllerinin elektronik enerji, $\mu$,

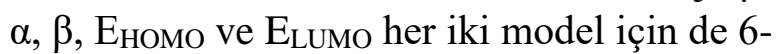
$311++G(d, p)$ taban seti kullanılarak hesaplandi. Hesaplamalar sonucundan elde edilen elektronik enerji, $\mu, \alpha, \beta$, ELumo -Eномо değerleri Tablo 2'de verildi. Ayrıca en yüksek dolu orbitaller (HOMO) ve en düşük boş orbitaler (LUMO)'nun 3D çizimi Şekil 4‘de verildi.

Tablo 2. I, II ve III moleküllerinin elektronik, HOMO, LUMO enerji, dipol moment, polarizebilite, hyperpolarizebilite, $\Delta \mathrm{Eg}$ değerleri.

B3LYP/6-311++G(d,p)

\begin{tabular}{|c|c|c|c|c|c|c|c|c|}
\hline \multicolumn{2}{|c|}{ Elektronik enerji (a.u) } & \multirow{2}{*}{$\begin{array}{l}\mu(\mathrm{D}) \\
1.1773\end{array}$} & \multirow{2}{*}{$\begin{array}{l}\alpha \text { (a.u) } \\
100.76\end{array}$} & \multirow{2}{*}{$\begin{array}{l}\beta \text { (a.u) } \\
214.04\end{array}$} & \multirow{2}{*}{$\begin{array}{l}\beta\left(10^{-30} \mathrm{esu}\right) \\
1.8492\end{array}$} & \multirow{2}{*}{$\begin{array}{l}\text { Еномо (a.u) } \\
-0.251076\end{array}$} & \multirow{2}{*}{$\begin{array}{l}\text { Elumo (a.u) } \\
-0.052534\end{array}$} & \multirow{2}{*}{$\begin{array}{l}\Delta \mathrm{Eg}_{\mathrm{g}} \\
5.4025\end{array}$} \\
\hline I & -539.01355687 & & & & & & & \\
\hline II & -638.29019337 & 1.1922 & 101.07 & 239.98 & 2.0733 & -0.251794 & -0.046100 & 5.5972 \\
\hline III & -998.63919214 & 1.2541 & 115.87 & 341.87 & 2.2935 & -0.255166 & -0.060316 & 5.3021 \\
\hline \multicolumn{9}{|c|}{$\mathrm{HF} / 6-311++\mathrm{G}(\mathrm{d}, \mathrm{p})$} \\
\hline I & -535.86514946 & 1.1707 & 90.03 & 157.16 & 1.3577 & -0.333340 & 0.038238 & 10.1110 \\
\hline II & -634.75559390 & 1.1633 & 89.25 & 164.34 & 1.4198 & -0.339872 & 0.038331 & 10.2913 \\
\hline III & -994.79072072 & 1.2953 & 102.42 & 199.80 & 1.7261 & -0.338813 & 0.038050 & 10.2549 \\
\hline
\end{tabular}

Moleküler sistemlerin hiperpolarizebilite değerinin değerlendirilmesi, üre molekülünün hiperpolarizabilite değeri referans alınarak yorumlanmaktadır (Sangeetha et al., 2014; Hadigheh-Rezvan et al., 2018; DemirKanmazalp, 2017; Turhan Irak and Gümüş 2017). Hesaplama sonuçlarına göre. I, II ve III moleküllerinin hiperpolarizebilite değerleri üre molekülünün hiperpolarizebilite değerinden yaklaşık olarak, sırasıyla B3LYP/6-311++G(d,p)' de 2.3698, 2.6570, 2.9392 kez daha büyük olduğu tespit edilmiştir. (B3LYP/6-311++G(d,p) için; üre $\beta=0.7803 \times 10^{-30}$ esu, 1 a.u. $=8,6393 \times 10^{-33}$ esu). 


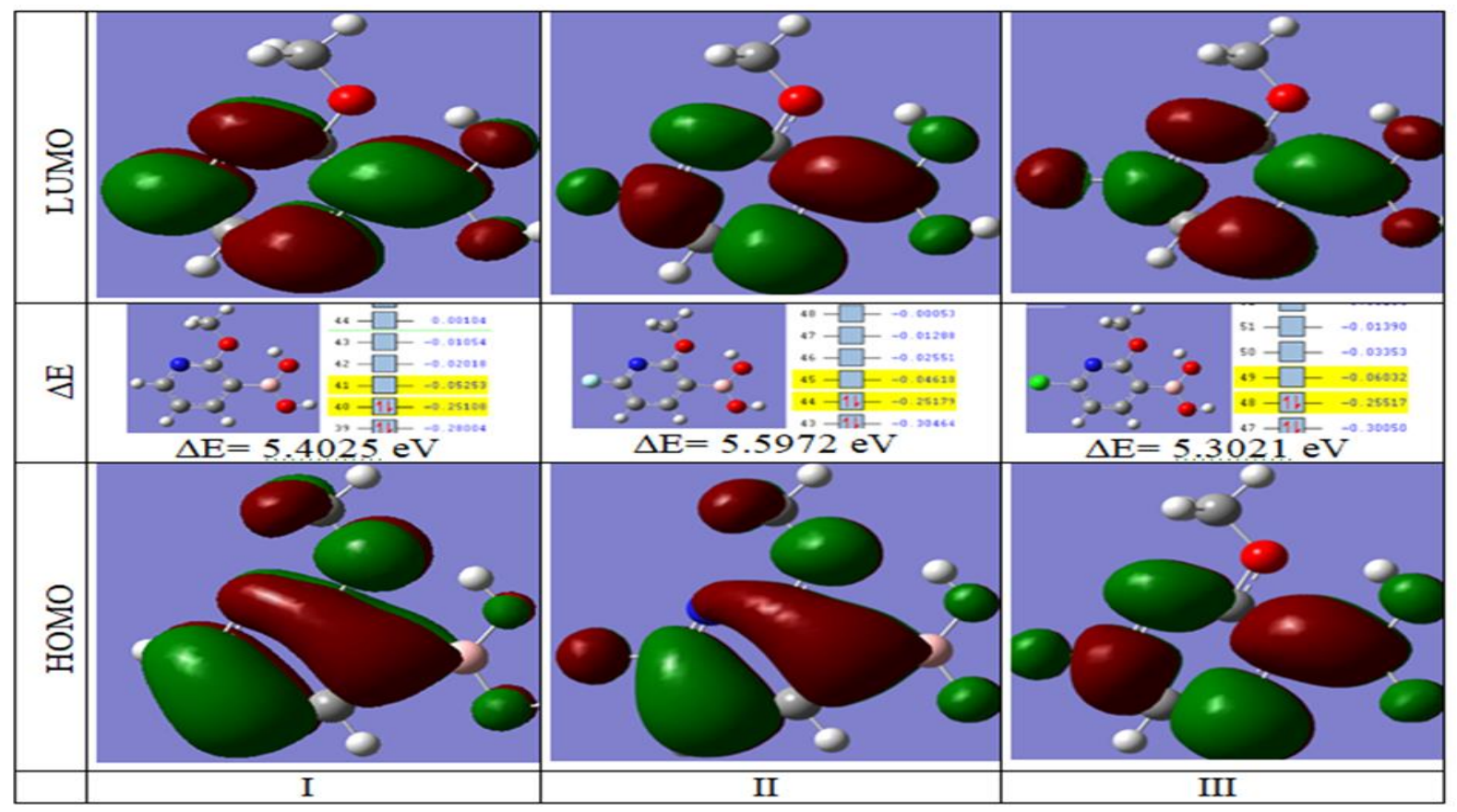

Şekil 5. I, II ve III moleküllerinin en yüksek dolu ve en düşük boş orbitallerinin üç boyutlu (3D) çizim.

\subsection{NMR ve IR Spektral Analiz}

Aromatik yapılarda bulunan karbon atomlarının NMR kimyasal kayma değerleri 100-150 ppm civarında görüldüğü iyi bilinmektedir (Pihlaja and Kleinpeter, 1994; Kalinowski et al., 1988). Fakat, yapıda bulunan elektronegatif atomlar, aromatik karbon atomlarının NMR sinyalleri daha yüksek değerlerde görülmesini sağlar. Çalışmamızda, 2-methoxypyridine-3-boronic acid ve 6- substitue türevlerinin (I, II, III) yapisinda bulunan piridin halkasindaki aromatik karbonlar B3LYP/6-311+G(2d,p) metodunda 174.39-112.751 ppm aralıklarında, $\mathrm{HF} / 6-31 \mathrm{G}$ (d) metodunda 163.41-93.91 ppm aralıklarında NMR sinyali verir ve bu sinyaller deneysel olarak, 166.06115.02 ppm aralıklarında görüldü. Piridin halkasında bulunan karbon atomlarının bu kadar düşük alanda çıkmasının sebebi elektronegatif $\mathrm{N}$ atomunun yapıda bulunmasından dolayıdır. Yapıda bulunan metoksi karbon atomları (C6) deneysel 53.78 B3LYP/6-311+G(2d,p) metodunda 54.52$55.63 \mathrm{ppm}$ aralıklarında, $\mathrm{HF} / 6-31 \mathrm{G}$ (d) metodunda ise 47.26-48.77 ppm aralıklarında sinyaller verir.

${ }^{1}$ H-NMR spektrumu için, aromatik protonlar (H3 ve H4) deneysel olarak 7.48 ppm'de, teorik olarak B3LYP'de 8.34-6.62 ppm aralıklarında ve HF'de ise 8.75-5.97 ppm aralıklarında NMR pikleri gözlenir. Çalışmamızda, metoksi grubu protonları (H5H7), deneysel olarak 3.88 ppm'de teorik olarak B3LYP/6-311+G(2d,p) metodunda 4.05-3.67 ppm aralıklarında, HF/6-31G (d) metodunda 3.69-3.47 ppm aralıklarında görülmüştür. Vileşiklerin NMR kimyasal kayma değerleri beklendiği bölgelerde görülmektedir. I, II ve III moleküllerinin ${ }^{13} \mathrm{C}$ ve ${ }^{1} \mathrm{H}$ NMR kimyasal kayma değerleri B3LYP/6-311+G(2d,p) ve HF/6-31G(d) yöntemleri ile hesaplandı ve sonuçlar Tablo 3 'de verildi. I, II ve III molekülleri 19 atoma sahip olduğundan dolayı düzlem içi ve diş1 olmak üzere 51 titreşim frekansına sahiptir. $\mathrm{Bu}$ çalışmada I, II ve III molekülünün titreşim frekansları B3LYP/6-311++G(d,p) ve $\mathrm{HF} / 6-311++\mathrm{G}(\mathrm{d}, \mathrm{p})$ teori düzeyinde hesaplatıld1. Her iki modelde hesaplatılan her titreşim modun, potansiyel enerji dağılımı VEDA programinda (Jamroz, 2004) yapılarak, işaretlenmeleri yapıldı. B3LYP/6$311++\mathrm{G}(\mathrm{d}, \mathrm{p})$ yöntemi ile elde edilen titreşim frekansları 0.983 skala faktörü (Sundaraganesana et al., 2005) ile çarpılarak uyumlu hale getirildi. Elde edilen titreşim modlarında seçilen bazı gerilme titreşimleri Tablo 4'de verildi. 
Tablo 3. I, II ve III moleküllerinin ${ }^{13} \mathrm{C}$ ve ${ }^{1} \mathrm{H}$ NMR kimyasal kayma değerleri.

\begin{tabular}{cccccccc}
\hline & \multicolumn{3}{c}{ B3LYP/6-311++G (2d, p) } & Deneysel & \multicolumn{3}{c}{ HF/6-31G (d) } \\
\hline Atom & $\mathrm{X}=\mathrm{H}(\mathrm{I})$ & $\mathrm{X}=\mathrm{F}(\mathrm{II})$ & $\mathrm{X}=\mathrm{Cl}(\mathrm{III})$ & III $^{\mathrm{b}}$ & $\mathrm{X}=\mathrm{H}(\mathrm{I})$ & $\mathrm{X}=\mathrm{F}(\mathrm{II})$ & $\mathrm{X}=\mathrm{Cl}(\mathrm{III})$ \\
\hline 1-C & 174.18 & 174.39 & 172.90 & 166.06 & 162.06 & 163.41 & 161.54 \\
5-C & 155.12 & 173.67 & 164.87 & 148.58 & 151.70 & 159.42 & 154.80 \\
3-C & 151.92 & 156.64 & 152.90 & 147.38 & 149.07 & 157.08 & 153.21 \\
4-C & 121.20 & 104.49 & 121.14 & 116.28 & 108.45 & 47.80 & 108.71 \\
2-C & 116.60 & 112.75 & 114.10 & 115.02 & 104.50 & 91.93 & 102.70 \\
6-C & 54.52 & 55.63 & 55.27 & 53.78 & 47.26 & 98.77 & 47.82 \\
\hline X-H & 8.40 & -- & -- & -- & 8.61 & -- & -- \\
4-H & 8.34 & 6.62 & 6.98 & 7.48 & 8.32 & 5.97 & 6.46 \\
3-H & 6.92 & 8.54 & 8.27 & 7.48 & 6.53 & 8.75 & 8.53 \\
2-H & 6.80 & 6.36 & 6.23 & 7.90 & 5.22 & 5.03 & 5.06 \\
7-H & 4.05 & 4.04 & 3.96 & 3.88 & 3.67 & 3.69 & 3.64 \\
5-H & 4.05 & 4.05 & 3.67 & 3.88 & 3.67 & 3.69 & 3.44 \\
6-H & 3.78 & 3.78 & 3.96 & 3.88 & 3.42 & 3.47 & 3.64 \\
1-H & 3.53 & 3.34 & 3.26 & 7.90 & 2.84 & 2.85 & 2.88 \\
\hline
\end{tabular}

Tablo 4. I, II ve III moleküllerinin seçilmiş dalga sayıları $\left(\mathrm{cm}^{-1}\right)$.

\begin{tabular}{|c|c|c|c|c|c|}
\hline \multicolumn{6}{|c|}{ B3LYP/6-311++G (d,p) } \\
\hline $\mathrm{X}=\mathrm{H}$ & İşaretlemeler & $\mathrm{X}=\mathrm{F}$ & İşaretlemeler & $\mathrm{X}=\mathrm{Cl}$ & İşaretlemeler \\
\hline 3687.08 & $v \mathrm{OH}(100)$ & 3686.88 & $v \mathrm{OH}(100)$ & 3687.16 & $v \mathrm{OH}(99)$ \\
\hline 3596.18 & $v \mathrm{OH}(99)$ & 3609.11 & $v \mathrm{OH}(100)$ & 3611.39 & $v \mathrm{OH}(99)$ \\
\hline 3064.27 & $v \mathrm{CH}(93)$ & 3085.27 & $v \mathrm{CH}(99)$ & 3083.80 & $v \mathrm{CH}(100)$ \\
\hline 3048.00 & $v \mathrm{CH}(95)$ & 3055.09 & $v \mathrm{CH}(100)$ & 3056.57 & $v \mathrm{CH}(100)$ \\
\hline 3022.02 & $v \mathrm{CH}(77)$ & -- & -- & -- & -- \\
\hline 3014.21 & $v \mathrm{CH}(95)$ & 3020.30 & $v \mathrm{CH}(94)$ & 3020.14 & $v \mathrm{CH}(94)$ \\
\hline 2992.05 & $v \mathrm{CH}(100)$ & 2996.51 & $v \mathrm{CH}(79)$ & 2995.97 & $v \mathrm{CH}(100)$ \\
\hline 2920.79 & $v \mathrm{CH}(95)$ & 2923.68 & $v \mathrm{CH}(94)$ & 2923.20 & $v \mathrm{CH}(94)$ \\
\hline 1557.95 & $v \mathrm{NC}(26) v \mathrm{CC}(28)$ & 1550,06 & $v \mathrm{NC}(26) v \mathrm{CC}(24)$ & 1563.23 & $v \mathrm{NC}(22) v \mathrm{CC}(46)$ \\
\hline
\end{tabular}

Genelde aromatik $\mathrm{C}-\mathrm{H}$ gerilme titreşimleri $3000-3100 \mathrm{~cm}^{-1}$ aralığında gözlenirken, $\mathrm{C}=\mathrm{C}$ gerilmesi $1600-1585 \mathrm{~cm}^{-1}$ ve $1500-1400 \mathrm{~cm}^{-1}$ bölgesinde görülür. Hesaplama sonuçlarında boronik asit grubunun $\mathrm{O}-\mathrm{H}$ gerilme titreşimleri $3596.18-3687.16 \mathrm{~cm}^{-1}$ bölgesinde gözlendi. Benzer moleküllerle yapılan deneysel ve teorik çalışmalarda değerler sırası ile 3343-3496 ve $3687-3722 \mathrm{~cm}^{-1}$ bölgesinde gözlenmiştir (Yalçın, 2015). Deneysel değerlere bu çalışmada gözlenen $\mathrm{O}-\mathrm{H}$ gerilme titreşimlerinin daha yakın olduğu görüldü. Metoksi grubunun ve piridin halkası üzerindeki C-H gerilme titreşimleri 2920.79-
$3020.14 \mathrm{~cm}^{-1}$ ve $3022.02-3083.80 \mathrm{~cm}^{-1}$ bölgesinde görüldü. Piridin halkası üzerindeki $\mathrm{C}-\mathrm{H}$ gerilme titreşimlerinin metoksi grubu C$\mathrm{H}$ gerilme titreşimlerininden daha yüksek bölgede gözlendi. Ayrıca flor ve klor substitüentlerinin molekülün titreşim frekanslarını etkiledikleri hesaplama sonuçlarında anlaşılmaktadır.

\section{Sonuç ve Tartışma}

2-metoksipiridin-3-boronik asit, onun flor ve klorlu substitüeli; 6-floro-2-metoksipiridin-3boronik 6-kloro-2-metoksipiridin-3-boronik asit moleküllerinin konformasyon analizi 
yapılarak, moleküllerin Hartree Fock metodu ve yoğunluk fonksiyoneli teorisi 6$311++\mathrm{G}(\mathrm{d}, \mathrm{p}) \quad$ temel seti kulanilarak hesapland. Bu moleküllerin denge durumu dipol momenti, polarizebilite, hiperpolarizebilite, titreşim frekansları, ${ }^{1} \mathrm{H}-\mathrm{ve}$ ${ }^{13} \mathrm{C}$-NMR kimyasal kayma değerleri, en yüksek dolu molekül orbital, en düşük boş molekül orbital enerjileri her iki yöntem ile hesaplandı. Bunlara ek olarak, hesaplanmış Еномо ve E Еuмо enerjileri kullanılarak molekülün sınır moleküler enerji aralığı elde edildi. Bu çalışmada, moleküllerin yapısal, elektronik ve lineer olmayan optik özellikleri teorik olarak belirlendi ve bu değerler daha önce yapılan deneysel değerlerle karşılaş̧ırılmakla birlikte flor ve klorlu sübstitüentlerini belirlenen büyüklükler üzerinde etkileri incelendi. Flor ve klor sübstitüentlerinin, molekülün bağ uzunluğu ve dihedral açılarını çok küçük oranda etkilemesine karşıık, bağ açılarını, bariyer yüksekliklerini ve titreşim frekanslarını daha fazla etkilediği görüldü. Ancak, sübstitüent etkilerinin dipol moment, polarizebilite, büyüklükleri önemli ölçüde etkilediği ve molekülün bu özelliklerinden hiperpolarizebilite değerinin büklüğ̈̈nün artması, molekülün lineer olmayan optik malzeme adayı olduğunu göstermektedir. 2metoksipiridin-3-boronik asit, 6-floro-2metoksipiridin-3-boronik ve 6-kloro-2metoksipiridin-3-boronik asit moleküllerinin hiperpolarizebilite değerleri, üre molekülünkinden sırası ile $2.3698,2.6570$, 2.9392 büyük olduğu belirlendi ve sıralaması III $>$ II $>\mathbf{I}$ şeklinde olmaktadir. Her iki metotta hesaplanan 2-metoksipiridin-3-boronik asidin yapisal parametreleri, literatürdeki verilerle karşılaştırıldı ve yapısal parametreler arasında iyi bir uyum olduğu görüldü.

\section{Kaynaklar}

Baker, S. J., Akama, T., Zhang, Y. K., Sauro, V., Pandit, C., Singh, R., Kully, M., Khan, J., Plattner, J. J., Benkovic, S. J., Lee, V., Maples, K. R. 2006. Identification of a Novel Boron- containing Antibacterial Agent (AN0128) with Anti-inflammatory Activity, for the Potential Treatment of Cutaneous Diseases. Bioorg. Med. Chem. Lett. 16, 5963-5967.

Becke, A. D. 1988. Density-functional exchange-energy approximation with correct asymptotic behavior. Physical Review A, 38(6), 3098-3100.

Becke, A. D, 1993. Density-functional thermochemistry 3 . The role of exact exchange. The Journal of Chemical Physics, 98 (7), 5648-5652.

Bouillon, A., Lancelot, J. C., Collot, V., Bovy, P. R., Rault, S. 2002. Synthesis of novel halopyridinylboronic acids and esters. Part 1: 6-Halopyridin-3-yl-boronic acids and estersTetrahedron 58, 28852890.

Cai, D., Larsen, R. D., Reider, P. J. 2002. Effective lithiation of 3-bromopyridine: synthesis of 3-pyridine boronic acid and variously 3-substituted pyridines Tetrahedron Lett., 43, 4285-4287.

Cannizzo, C., Amigoni-Gerbier, S., Larpent, C. 2005. Boronic Acid-functionalized Nanoparticles: Synthesis by Microemulsion Polymerization and Application as a Re-usable Optical Nanosensor for Carbohydrates, Polymer, 46, 1269-1276.

Cooper, C. R., Spencer, N., James, T. D. 1998. Selective Fluorescence Detection of Fluoride Using Boronic Acids. Chem. Commun. 1365-1366.

Dabrowski, M., Lulinski, S., Serwatowski J., Szczerbinska, M. 2006. (2-Methoxy-3pyridyl) boronic acid, Acta. Crystallogr., C62, 702-704.

Demir-Kanmazalp, S. 2017. Investigation of Theoretical Calculations of 2-(1Phenylethylideneamino) Guanidine Compound: NBO, NLO, HOMOLUMO and MEP Analysis by DFT 
Method. Karaelmas Fen ve Müh. Dergisi, 7(2), 491-496.

Dennington, R., Keith, T., Millam, J. 2009. Semichem Inc., Gauss View, Version 5, Shawnee Mission KS,

Fragoso-Medina, A.J., Escobedo-Gonzalez, R. G., Nicolas-Vazquez, M.I., ArroyoRazo, G. A., Noguez-Cordova, M.O., Miranda-Ruvalcaba, R. 2017. A DFT Study of the Geometrical, Spectroscopical and Reactivity Properties of DiindolylmethanePhenylboronic Acid Hybrids. Molecules, 22, 1-25.

Fischer, F. C., Havinga, E. 1974. Thermal and photoinduced deboronations of some pyridine- and benzeneboronate anions. Recueil, 93, 21-24.

Fischer, F. C., Havinga, E. 1965. Pyridineboronic Acids, Recueil 84, 439440.

Frankland, E., Duppa, B. F. 1860. "Vorläufige Notiz über Boräthyl" Justus Liebigs Ann Chem 115, 319-322.

Frisch, M. J., Trucks, G. W., Schlegel, H. B., Scuseria, G. E., Robb, M. A., Cheeseman, J. R., Scalmani, G., Barone, V., Mennucci, B., Petersson, G. A., Nakatsuji, H., Caricato, M., Li, X., Hratchian, H. P., Izmaylov, A. F., Bloino, J., Zheng, G., Sonnenberg, J. L., Hada, M., Ehara, M., Toyota, K., Fukuda, R., Hasegawa, J., Ishida, M., Nakajima, T., Honda, Y., Kitao, O., Nakai, H., Vreven, T., Montgomery, J. A., Vreven, T. J., Peralta, J. E., Ogliaro, F., Bearpark, M., Heyd, J. J., Brothers, E., Kudin, N., Staroverov, V. N., Kobayashi, R., Normand, J., Raghavachari, K., Rendell, A., Burant, J. C., Iyengar, S. S., Tomasi, J., Cossi, M., Rega, N., Millam, J. M., Klene, M., Knox, J. E., Cross, J. B., Bakken, V., Adamo, C., Jaramillo, J., Gomperts, R., Stratmann, R. E., Yazyev, O., Austin, A.
J., Cammi, R., Pomelli, C. J., Ochterski, W., Martin, L. R., Morokuma, K., Zakrzewski, V. G., Voth, G. A., Salvador, P., Dannenberg, J. J., Dapprich, S., Daniels, A. D., Farkas, O., Foresman, J. B., Ortiz, J. V., Cioslowski, J., Fox, D. J., 2009. Gaussian Inc., (Wallingford, CT).

Hadigheh-Rezvan, V., Pilevar-Maleki, B. 2018, Structural and Optical Properties of Some 5, 8-Diaminoquinoxaline Schiff Bases:Quantum Chemical Calculations. Der Chemica Sinica, 9(1), 544-554.

Halo, T. L., Appelbaum, J., Hobert, E. M., Balkin, D. M., Schepartz, A. 2009. Selective Recognition of Protein Tetraserine Motifs with a Cellpermeable, Pro-fluorescent Bis-boronic Acid. J. Am. Chem.Soc. 131, 438-439.

Jabbour, A., Steinberg, D., Dembitsky, V. M., Moussaieff, A., Zaks, B., Srebnik, M. 2004. Synthesis and Evaluation of Oxazaborolidines for Antibacterial Activity against Streptococcus Mutans, J. Med.Chem., 47, 2409-2410.

Jamróz, M. H. 2004. Vibrational Energy Distribution Analysis: VEDA 4 program, Warsaw

Kalinowski, H. O., Berger, S., Braun, S. 1988. Carbon-13 NMR Spectroscopy, John Wiley \& Sons, Chichester.

Krishnan, R., Binkley, J. S., Seeger, R., Pople, J. A. 1980. Self-consistent molecularorbital methods. basis set for correlated wave-functions, The Journal of Chemical Physics, 72, 650-654.

Lee C. T, Yang W. T, Parr R. G. 1988. Development of the colle-salvetti correlation-energy formula into a functional of the electron density. Physical Review B, 37, 785-789.

McLean, A. D., Chandler, G. S. 1980. Contracted Gaussian basis sets for 
molecular calculations. I. Second row atoms, $\mathrm{Z}=11-18$. The Journal of Chemical Physics, 72, 5639-5648.

Moller, C., Plesset, M. S. 1934. Note on an approximation treatment for manyelectron systems. Physical Review, 46, 618-622.

Petasis, N. A. 2007. Expanding Roles For Organoboron Compounds - Versatile And Valuable Molecules For Synthetic, Biological And Medicinal Chemistry Australian Journal of Chemistry, 60(11), 795-798.

Pihlaja, K., Kleinpeter, E. 1994. Carbon-13 NMR Chemical Shifts in Structural and Sterochemical Analysis, VCH Publishers, Deerfield, Beach.

Sangeetha, C. C., Madivanane, R., Pouchaname, V., Vijaya Prasath, R., 2014. Experimental (FT-IR \& FTRaman) and theoretical investigation, electronic properties of quinoxaline. International Journal of ChemTech Research, 6 (5), 2854-2865.

Saygıll, N. 2011. New pyridinylboronic acid and new heterobiaryls via CrossCoupling reaction of pyridinylboronic acids with heteroaryl halides ,Hacettepe University Journal of the Faculty of Pharmacy, 31, 85-96.

Sundaraganesana, N., Ilakiamania, S., Saleema, H., Wojciechowskib, P. M., Michalskab, D. 2005. FT-Raman and FT-IR spectra, vibrational assignments and density functional studies of 5bromo-2-nitropyridine, Spectrochimica Acta Part A, 61, 2995-3001.

Turhan Irak Z, Gümüș S, 2017. Heterotricyclic compounds via click reaction: A computational study. Noble International Journal of Scientific Research, 1(7), 80-89.

Yang, W., Fan, H., Gao, S., Gao, X., Ni, W., Karnati, V., Hooks, W. B., Carson, J.,
Weston, B., Wang, B. 2004. The First Fluorescent Diboronic Acid Sensor Specific for Hepatocellular Carcinoma Cells Expressing Sialyl Lewis X. Chem. Biol., 11, 439-448.

Yalçın, Y. 2015. The Theoretical and Experimental Investigation of the Vibrational Spectra of 4(Methylsulfanyl)Phenylboronic Acid Molecule. M. Sc. Thesis, Nevşehir Hacı Bektaş Veli Unıversıty, İnstitute of Science, Nevșehir.

Yang, W, Gao, X., Wang, B. 2003. Boronic acid compounds potential pharmaceutical Agents, Med. Res. Rev., 23, 346-368.

Varughese, S., Sinha, S. B., Desiraju, G. R. 2011. Phenylboronic acids in crystal engineering: Utility of the energetically unfavorable syn,syn-conformation in co-crystal design,Sci china chem., 54 (12), 1909-1919. 